\title{
CHARGE EFFECT SOLITONS
}

\author{
E. BEN-JACOB ${ }^{\text {a,b }}$, K. MULLEN ${ }^{\text {a }}$ and M. AMMAN ${ }^{a}$ \\ a Department of Physics, University of Michigan, Ann Arbor, MI 48109, USA \\ b School of Physics and Astronomy, Raymond and Beverly Sackler Faculty of Exact Sciences, Tel-Aviv University, \\ 69978 Tel-Aviv, Israel
}

Received 24 May 1988; revised manuscript received 27 December 1988; accepted for publication 3 January 1989 Communicated by A.A. Maradudin

\begin{abstract}
We present an effective circuit model of a chain of mesoscopic tunnel junctions. We show that the system possesses a soliton solution. The propagation of the soliton corresponds to the tunneling of a single charge along the system. We study the properties of this charged soliton and calculate the resulting $I-V$ characteristics. We discuss possible experimental realizations of the model and its relevance to other systems.
\end{abstract}

The electrostatic charging energy is now recognized as playing a crucial role in the dynamics of mesoscopic tunnel junctions, both in theory [1-15] and experiment [16-24]. Given a normal (i.e. non-superconducting), current driven junction with a capacitance $C$, it is energetically unfavorable for an electron to tunnel when the voltage across the junction is smaller than $e / 2 C$ provided that the capacitance is sufficiently small so that $e^{2} / 2 C>k_{\mathrm{B}} T$, where $T$ is the temperature, and that the junction has a resistance $R>\hbar / 4 e^{2}$, so that the electrons are localized on either side of the barrier. Various model predict that the application of a current source of magnitude $I_{\mathrm{dc}}$ to such a junction will produce an "inverse Josephson effect": oscillations in the voltage with an amplitude of $e / 2 C$, and a frequency $I_{\mathrm{dc}} / e$ for $I_{\mathrm{dc}}<$ $e / R C$. At large currents $\left(I_{\mathrm{dc}}>e / R C\right)$ the average voltage will be shifted above the ohmic result by $e /$ $2 C$. In addition, related effects such as the "Coulomb staircase" (steps in the $I-V$ characteristic) have been predicted [10] and observed in experiments on two mesoscopic junctions connected in series [2022]. Similar oscillations are predicted in ultrasmall capacitance Josephson junctions, but with an amplitude of $e / C$ and a frequency $1 / 2 e$.

From their inception, it was realized that the above oscillations are reminiscent of the ac Josephson effect. As has been pointed out elsewhere [13,15,24], there is a strong analogy between the dynamics due to charging in mesoscopic normal tunnel junctions and those in ordinary Josephson junctions [25] $]^{\# 1}$. In this Letter we pursue this analogy to develop an effective equation $[25,26]$ to describe the dynamics of a single normal mesoscopic tunnel junction. Using this effective equation we study the soliton dynamics of a chain of serially coupled junctions. These charge effect solitons are associated with the propagation of a charge along the chain. One realization of such a chain is a narrow strip of conducting grains capacitively coupled through the supporting substrate. The propagation of the soliton is associated with the tunneling of a single electron at a time between two grains. Below we calculate some of the properties of such solitons and make predictions about possible experiments. We also extend our analysis to the superconducting case and discuss the possibility of dissipationless transport among the grains arising from the motion of the solitons.

We start by discussing an effective circuit description for a mesoscopic normal tunnel junction driven by an external current source. At zero temperature the tunneling rate of electrons as a function of the

\footnotetext{
\#1 An effective equation for the case of ultrasmall capacitance Josephson junctions, derived from a different starting point, can be found in ref. [26].
} 
voltage on the junction is zero up to a voltage $e / 2 C$, and then increases linearly thereafter $[4,5,10,13]$. (This implicitly assumes that $R>\hbar / 4 e^{2}$ so that the electron is localized on one half of the junction or the other.) At low values of the driving force $\left(I_{\mathrm{dc}} \rightarrow 0\right)$ the junction repeatedly charges up to a voltage $e / 2 C$ and an electron then tunnels. This cycle produces a sawtooth oscillation in the voltage with an average of zero. Such a process can be expressed as a nonlinear capacitance, $f(Q)$, which expresses the voltage on the junction as a function of $Q$, the amount of charge brought in from the external source,

$f(Q)=\left[\left(Q+\frac{1}{2} e\right) \bmod e-\frac{1}{2} e\right] / C$,

$Q(t) \equiv \int^{t} I\left(t^{\prime}\right) \mathrm{d} t^{\prime}$

(see fig. 1a). Note that $Q$ is the total charge delivered by the external source, not the actual charge on the junction. For finite values of the current the junction will charge up to a voltage $V>e / 2 C$ before an electron will tunnel. The voltage as a function of time
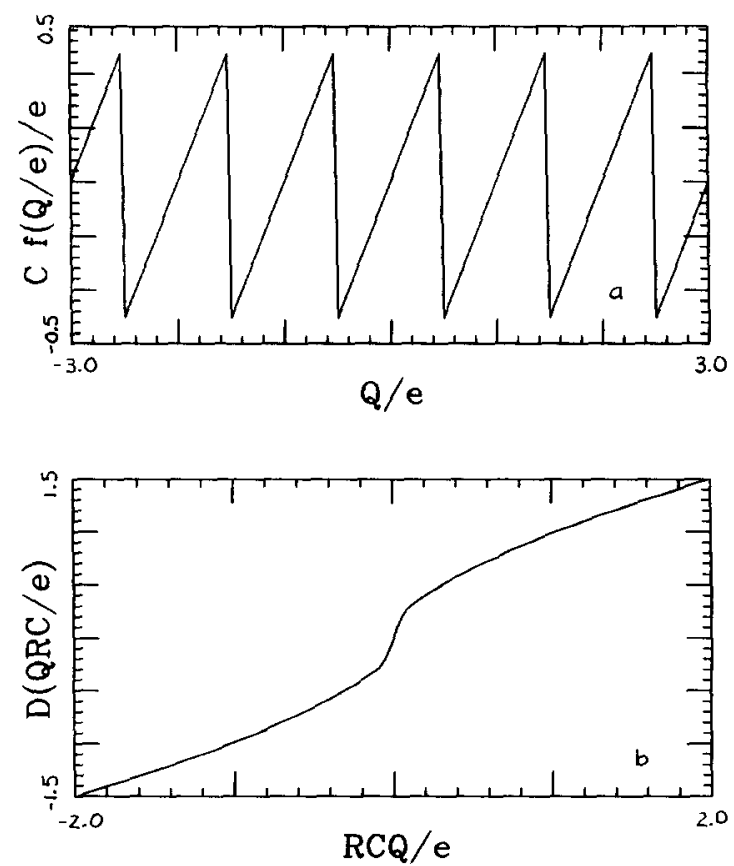

Fig. 1. The mesoscopic tunnel junction is represented by a nonlinear capacitance $f(Q)$ and a non-linear dissipation $D(\dot{Q})$. (a) A plot of $C f(Q / e) / e$, as given in eq. (1). (b) A plot of $D(R C \dot{Q} /$ $e$ ), whose asymptotic behavior is given by eq. (2). will oscillate (with some noise) about an average value determined by a competition between the continuous charging due to the external source and the stochastic discharging of the tunneling current. Once the tunneling rate is known it is possible to calculate the average voltage on the junction as a function of the current $[5,12]$, which we denote by the function $D(\dot{Q})$, plotted in fig. $1 \mathrm{~b}$. The asymptotic behavior of $D(\dot{Q})$ is given by

$$
\begin{aligned}
& D(\dot{Q}) \approx \operatorname{sgn}(\dot{Q}) \sqrt{\pi e R|\dot{Q}| / 2 C}, \text { for }|\dot{Q}|<e / 2 R C, \\
& \approx \operatorname{sgn}(\dot{Q})(R|\dot{Q}|+e / 2 C), \text { for }|\dot{Q}|>e / 2 R C \text {. }
\end{aligned}
$$

We can then view the voltage drop across the mesoscopic tunnel junction as arising from a series combination of three voltage drops, across a non-linear capacitor $f(Q)$, a non-linear resistor, $D(\dot{Q})$, and a noise source $\sigma(\dot{Q})$ reflecting the stochastic fluctuations of the voltage about $D(\dot{Q})$. This serially resistive junction $[25,26]$ (SRJ) model is an analog of the resistively shunted junction (RSJ) model [27] used to describe a Josephson junction (for a review of the RSJ model in Josephson junctions see, e.g., ref. [28]).

Since the device is modelled in terms of voltage drops it is more natural to consider a voltage source rather than a current source. The equation of motion for the SRJ model is then derived by equating the sum of the voltage drops across each element to an applied voltage $V$ (fig. 2a). Although the charge on the junction changes discontinuously as electrons tunnel, $Q$ must be a continuous function due to the finite response time of the source. We express this delay time by a phenomenological inductance $L$. We assume that this delay time is longer than the time it takes an electron to tunnel [29].

$$
L \ddot{Q}+D(\dot{Q})+f(Q)=V,
$$

where we have neglected the noise term $\sigma(\dot{Q})$ and have neglected a phase shift in $f(Q)$ that depends upon $\dot{Q}$. Rigorous derivations of the precise form of

\#2 The asymptotic expansions in eq. (2) are not equal at $\dot{Q}=e /$ $2 R C$. Calculations were done using an interpolating formula between the two expressions. The interpolation was chosen to be in agreement with the results of stochastic simulations of a single junction driven by a current source. These comments also apply to similar, subsequent expressions for the dissipation. 


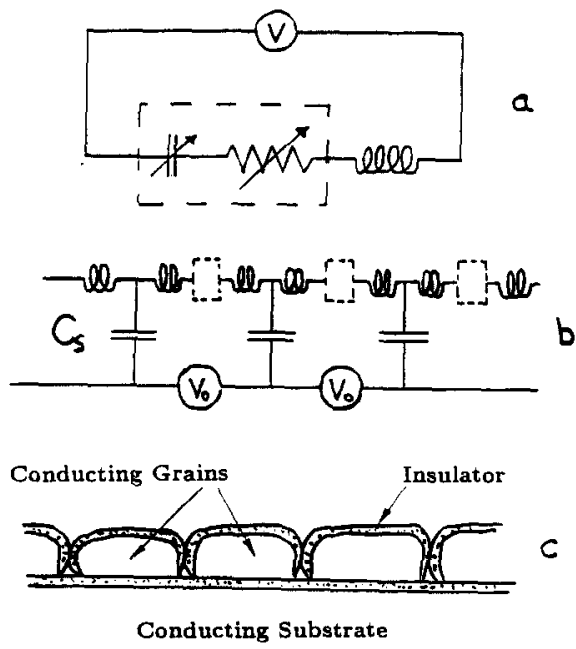

Fig. 2. (a) The effective circuit diagram of a mesoscopic tunnel junction. The junction is represented as a series combination of a non-linear resistor and a non-linear capacitor (dashed box). The external circuit must be represented by the appropriate standard electrical elements. Here we have included an inductor to represent the finite rearrangement time of the charges. (b) A schematic showing a chain of coupled mesoscopic tunnel junctions. Each junction is also capacitively coupled to a common underlying substrate through a capacitor $C_{\mathrm{s}}$ and is driven by a potential difference $V_{0}$. (c) One possible realization of such a system is a chain of small conducting grains, each separated by an insulating layer that serves as a tunneling barrier. A voltage can be applied across the substrate to enforce the potential difference $V_{0}$.

$f(Q)$ and $D(\dot{Q})$ can be obtained from a master equation analysis of the stochastic process that describes the junction dynamics. However, we do not expect any qualitative difference in the results of our approximation and those of the exact expressions. Simulations have shown that eq. (3) is in good agreement with the stochastic process that it models.

In employing eq. (3) we have to properly account for the character of the driving circuit (i.e. voltage source, current source, etc.) by introducing additional terms that depend upon $Q$ or its derivatives. For example, to calculate the response of the junction to an external current source, it is necessary to add a term corresponding to a large external resistance, $R_{e x} \dot{Q}$. Alternatively, if the junction is made from superconducting materials then we would expect the form of $f(Q)$ and $D(\dot{Q})$ to be modified. A superconducting junction will have a dissipation $D(\dot{Q})$ that reflects the gap in the quasiparticle states.
In this case $f(Q)$ will still be a sawtooth for $E_{\mathrm{J}} /$ $E_{\mathrm{C}} \ll 1$ where $E_{\mathrm{J}}$ is the Josephson coupling energy, but will become more rounded for $E_{\mathrm{J}} / E_{\mathrm{C}} \ll 1$. A more detailed discussion of the SRJ model and its variations will be given in refs. $[25,26]$.

The advantage of such a lumped circuit model is that complicated configurations of junctions can be broken down into a set of simple elements. It is now straightforward to apply all the studies of coupled Josephson junctions. For example, we consider two identical mesoscopic junctions in series with a capacitor $C_{\mathrm{s}}$ connected between the interjunction region and ground, in analogy to the inductively coupled Josephson junctions in a dc SQUID. The extension of eq. (1) to two identical junctions is:

$$
\begin{aligned}
& L \ddot{Q}_{1}+D\left(Q_{1}\right)+f\left(Q_{1}\right)=\frac{1}{2} V-\frac{Q_{1}-Q_{2}+Q_{0}}{C_{\mathrm{s}}}, \\
& L \ddot{Q}_{2}+D\left(Q_{2}\right)+f\left(Q_{2}\right)=\frac{1}{2} V+\frac{Q_{1}-Q_{2}+Q_{0}}{C_{s}},
\end{aligned}
$$

where $Q_{1}$ and $Q_{2}$ are the charges transferred to the first and second junctions respectively, $Q_{0}$ is the initial charge imbalance between the two junctions. The quantity $Q_{1}-Q_{2}+Q_{0}$ is charge stored between the two junctions on the coupling capacitor $C_{\mathrm{s}}$. This coupling capacitor introduces coherence to the dynamics of the tow junctions. Analysis of eq. (4) demonstrates both a running solution, in which the two junctions transfer charge simultaneously, and a beating solution, in which the junctions alternately transfer charge. Such solutions have been studied in great detail in Josephson junctions [30].

Using this SRJ model we can generalize eq. (4) to an effective equation of motion for a chain of serially coupled junctions, in analogy with the sine-Gordon equation for a long Josephson junction $[31,32]$. In fig. $2 \mathrm{~b}$ we show a schematic of such a system, and in fig. $2 \mathrm{c}$ one possible realization. The equation of motion for $Q_{i}$, the charge brought to the $i$ th junction is

$L \ddot{Q}_{i}+D\left(\dot{Q}_{i}\right)+f\left(Q_{i}\right)=V_{i}+\frac{Q_{i+1}+Q_{i-1}-2 Q_{i}}{C_{s}}$,

where $V_{i}$ is the externally imposed voltage difference between the two capacitors, and $C_{\mathrm{s}}$ is the capacitive coupling to the substrate. This is obtained by equating the sum of the voltage drops between the upper 
and lower halves of the $i$ th current loop. This is very similar to the discrete version of the sine-Gordon equation with the linear "mod" function $f(Q)$ replacing the sine function, and can be referred to as a "line-Gordon" equation. In the strong coupling limit where $C_{\mathrm{s}} \ll C$ (the number of junctions within a stationary soliton scales as $\sqrt{C / C_{\mathrm{s}}}$ ) we can approximate the difference in eq. (5) by a derivative to obtain

$L \ddot{Q}+D(\dot{Q})+f(Q)=V(x)+\frac{\Delta x^{2}}{C_{\mathrm{s}}} \frac{\partial^{2} Q}{\partial x^{2}}$,

where $\Delta x$ is the distance between junctions (i.e. the size of the grains in fig. $2 \mathrm{c}$ ). If we measure time in units of $\sqrt{L C}$ (the delay time within a grain), voltage in units of $e / C$, energy in $e^{2} / C$, current in units of $e / R C$, distance in units of $\Delta x \sqrt{C / C_{s}}$, velocity in units of $\Delta x / \sqrt{L C_{\mathrm{s}}}$ (which plays the role of the "speed of light" for the system), we obtain the following dimensionless equation of motion for the variable $q \equiv Q / e$,

$\ddot{q}-q_{x x}+d(\dot{q})+f(q)=V(x)$,

where

$$
\begin{aligned}
d(\dot{q}) & =\operatorname{sgn}(\dot{q}) \sqrt{\frac{1}{2} \pi \kappa \mid \dot{q}} \mid, & & \text { for }|\dot{q}|<1 / 2 \kappa, \\
& =\operatorname{sgn}(\dot{q})\left(\kappa|\dot{q}|+\frac{1}{2}\right), & & \text { for }|\dot{q}|>1 / 2 \kappa,
\end{aligned}
$$

and

$f(q)=\left(q+\frac{1}{2}\right) \bmod 1-\frac{1}{2}$

and $\kappa=R C / \sqrt{L C}$. The parameter $\kappa$ plays the role of dissipation, and unlike the Josephson junction case the dissipation is proportional to the resistance.

We are now in principle in a position to apply all the studies developed to understand soliton solutions for the sine-Gordon equation. Below we give a few results aimed at comparison with experiments. In order to look for travelling wave solutions we rewrite eq. (6) in terms of $\eta=(x-v t) \gamma$ with $\gamma=1 /$ $\sqrt{1-v^{2}}$ to obtain

$q_{\eta \eta}+d\left(\nu \gamma q_{\eta}\right)=f(q)-V_{0}$,

where we have assumed $V(x)$ to be a constant, $V_{0}$. Note that this equation is the same as the equation of a single junction with $\kappa$ replaced by $\bar{\kappa}=v \gamma \kappa$, moving in the inverted potential, $F(q)=\int\left[f(q)-V_{0}\right] \mathrm{d} q$. We first look or a single freely propagating soliton solution, in the absence of driving force and dissipation. This soliton solution is the trajectory connecting the points $q(-\infty)=0$ and $q(\infty)=1$, which is given by

$$
\begin{aligned}
q(\eta) & =\frac{1}{2} \mathrm{e}^{\eta}, & & \text { for } \eta<0, \\
& =1-\frac{1}{2} \mathrm{e}^{-\eta}, & & \text { for } \eta>0 .
\end{aligned}
$$

In fig. 3 we show a plot of the charge effect soliton. The passage of the soliton front through a given junction in the chain corresponds to the transfer of exactly one electron across the junction. In other words the motion of the soliton corresponds to the propagation of a single charge along the chain. The center of the soliton corresponds to the grain at which the surplus electron resides. This charge effect soliton neatly complements solitons found in long Josephson junctions, which correspond to the motion of quantized bundles of magnetic flux.

Although the electron is confined to one grain at each time its effect is spread out over the soliton width which can be much larger than a single grain.
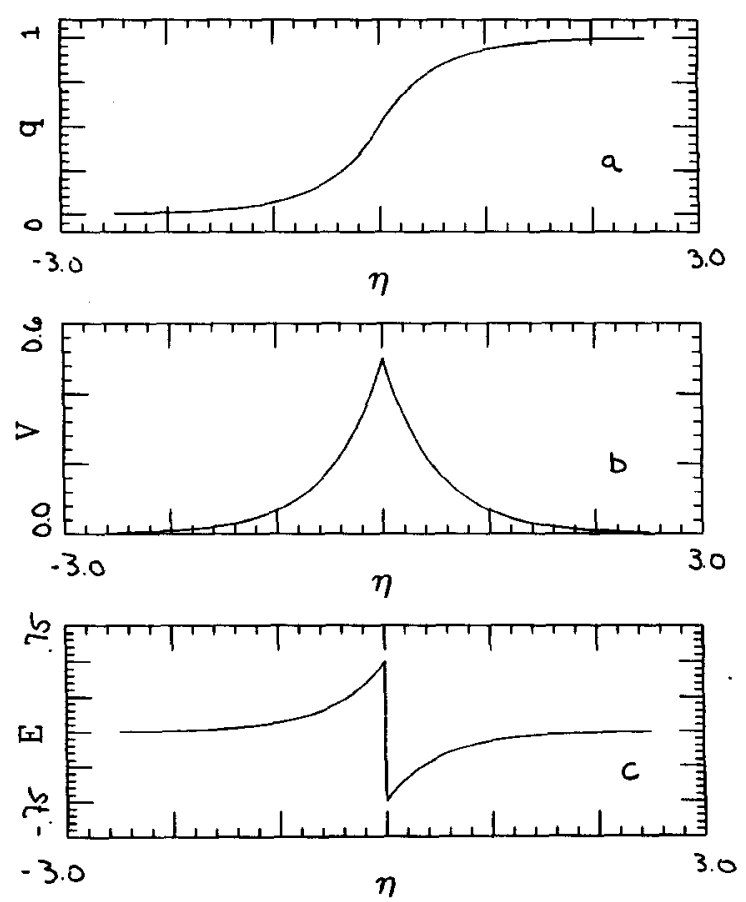

Fig. 3. (a) A plot of the charge effect soliton $q(\eta)$, as given in eq. (11). (b) The voltage $\left(q_{\eta}\right)$ as a function of position. The peak corresponds to the location of the droplet carrying the single excess charge. (c) The field $\left(q_{\eta \eta}\right)$ as a function of position. 
The dynamics of this "dressed" electron is the dynamics of the soliton itself. It is therefore useful to define collective coordinates for the soliton position and conjugate momentum [33]. These will have the simple interpretation as the position and momentum of the "dressed" electron. Following ref. [33] we define

$$
\begin{aligned}
& X \equiv \int_{-\infty}^{\infty} x q_{x} \mathrm{~d} x, \\
& P \equiv \int_{-\infty}^{\infty} \dot{q} q_{x} \mathrm{~d} x,
\end{aligned}
$$

where $q_{x}$ serves as the "weight function" of the soliton. It is straightforward to show that the Poisson bracket $\{P, Q\}=1$. For the freely propagating soliton with velocity $v$ we have that

$\dot{X}=v, \quad P=\frac{1}{4} \gamma v \equiv m_{0} \gamma v$.

This demonstrates that the "dressed" electron acts like a relativistic particle with a rest mass of $\frac{1}{4}$ in dimensionless units (or $\frac{1}{4} e^{2} L \sqrt{C_{\mathrm{s}} / C}$ with the proper dimensions) and an energy of $E=m_{0} \gamma=\frac{1}{4} \mathrm{e}^{2} \gamma /$ $\sqrt{C C_{\mathrm{s}}}$. Up to this point we have neglected the dissipation and the driving force. In the presence of dissipation for each value of the driving force there will be a unique steady state velocity. To calculate the velocity it can be shown that $\dot{P}=V_{0}-D(P)$ where $D(P)$ is

$$
\begin{aligned}
D(P) & =2 \operatorname{sgn}(P) \sqrt{\pi \kappa|P|} / 3, & & \text { for }|P|<1 / 2 \kappa, \\
& =\operatorname{sgn}(P)\left(\kappa|P|-\frac{1}{2}\right), & & \text { for }|P|>1 / 2 \kappa .
\end{aligned}
$$

In steady state we have that $D(P)=V_{0}$, which allows us to express $P$ as a function of $V_{0}$. To calculate the velocity in the underdamped limit $(\bar{\kappa}<1$ or $\gamma v<1 /$ $\kappa$, which yields $v<1 / \sqrt{1+\kappa^{2}}$ ) we can use the freely propagating soliton approximation $\left(P \approx \frac{1}{4} \gamma v\right)$, and find $v\left(V_{0}\right)$,

$$
\begin{aligned}
v=\frac{V_{0}^{2}}{\sqrt{\frac{1}{8 !} \pi^{2} \kappa^{2}+V_{0}^{4}}} \approx \frac{9 V_{0}^{2}}{\pi \kappa}, \text { for } V_{0} \ll \frac{1}{3} \sqrt{\pi \kappa}, \\
\approx 1, \quad \text { for } V_{0} \gg \frac{1}{3} \sqrt{\pi \kappa} .
\end{aligned}
$$

This result is valid in the underdamped limit $\kappa<1$ and $V_{0}<\sqrt{\frac{1}{9} \pi}$. We note that it is not possible to have a single soliton solution for $V_{0}>\frac{1}{2}$ since above this point the potential $F(q)$ has no minima.

Using the above we can directly calculate the $I-V$ characteristic of the system as was done for Josephson junctions in ref. [34]. The current through the chain in units of $e / R C$ is given by $I=e v \kappa / N$ where $N$ is the number of grains between two solitons in the chain that are spaced sufficiently far apart that they can be considered as independent. In fig. 4 we show the predicted single soliton branch of the $I-V$ characteristic for different values of $\kappa$. For the same $V_{0}$ the two soliton solution carries twice the current but with a larger dissipation. The $I-V$ characteristic for $\kappa<1$ will therefore consist of a series of branches, unequally spaced due to the nonlinearity in $D(Q)$, similar to those of fig. 4. This limit corresponds to the case where the charge relaxation time within the grain is longer than the $R C$ time. Such a value of $\kappa$ might be achieved by making the grains from a semiconductor with a long response time.

The framework developed above may be adopted to describe many other systems. One example is that

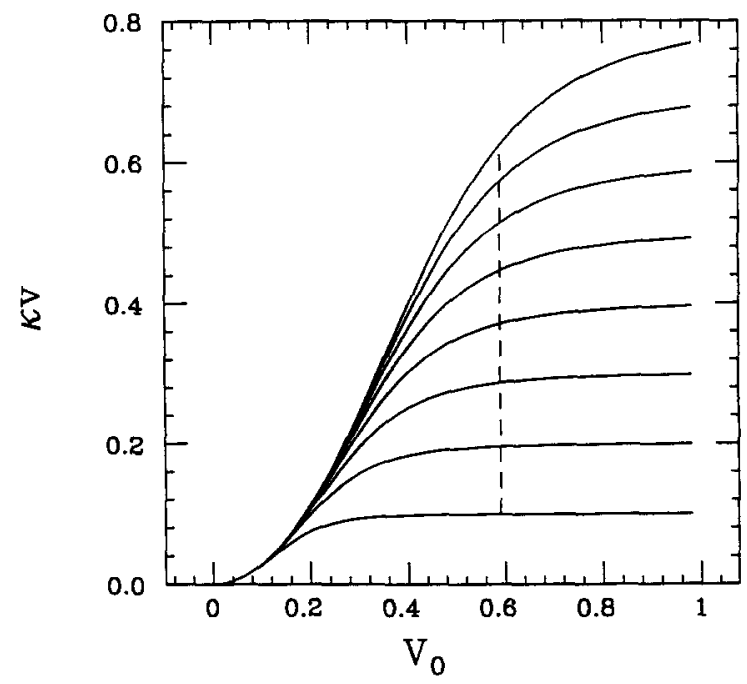

Fig. 4. A plot of the soliton velocity $v$ versus the applied voltage $V_{0}$ for $\kappa=0.1$ to $\kappa=0.8$ in steps of 0.10 , as given by eq. (14), Each line represents the current carried by the single soliton branch in the $I-V$ characteristic for a given value of $\kappa$. For fixed $\kappa$ there will be multiple branches in the $I-V$ characteristic corresponding to the two, three, etc., soliton solutions. These branches will be spaced unequally due to the non-linearity of the dissipation. 
of charge density wave ( $\mathrm{CDW}$ ) propagation where a phenomenological equation analogous to eq. (1) has been proposed $[35,36]$. Our picture may provide a link between this phenomenological model and the tunneling model [37] for CDW. Another example is a chain of serially connected mesoscopic Josephson junctions. It has been predicted that a single Josephson junction should show Bloch oscillations $[2,5-9,38]$ due to pair tunneling similar to the single electron oscillations. An equation similar to the SRJ model can be readily derived for the Josephson junction case. This case differs from the normal junction case in that as long as the motion of the soliton is slow, it can travel without dissipation. By "slow" we mean that the current carried by the soliton is less than the "Zener current" [38], which is given by the Josephson current times the ratio of the charging energy of a pair to the Josephson coupling energy. Since the longitudinal coupling of the pairs is through the substrate, the latter too must be superconducting for the soliton to move without dissipation. This provides a direct test of the existence of such dissipationless solitons. If they do exist they might provide a possible soliton mechanism to describe pair condensation in two dimensions in systems where the pairs are localized in space and propagate via tunneling between sites.

Here we have discussed only a few of the effects observable from charge effect solitons. Many others of the effects predicted for solitons in long Josephson junctions have their counterpart in solitons in a chain of mesoscopic normal tunnel junctions, as well as possible new effects. Their future study is made much simpler by the use of the SRJ model given here.

We have profited from many useful discussions with B. Orr, and G. Schön, and Z. Schuss. This research was partially supported by NSF Grant DMR 8608305, Grant DAAL 03-87-k-0007 from the Army Research Office. E.B.-J. is a Bat-Sheva Fellow. K.M. is supported by a fellowship from the Center for High Frequency Micro-electronics at the University of Michigan, and M.A. is supported by a Regent's Fellowship from the State of Michigan.

\section{References}

[1] T.L. Ho, Phys. Rev. Lett. 51 (1983) 2060; E. Ben-Jacob, E. Mottola and G. Schön, Phys. Rev. Lett. 51 (1983) 2064.

[2] E. Ben-Jacob and Y. Gefen, Phys. Lett. A 108 (1985) 289.

[3] E. Ben-Jacob, Y. Gefen, K. Mullen and Z. Schuss, in: SQUID 85 , eds. H.D. Hahlbohm and H. Lübbig (De Gruyter, Berlin, 1985).

[4] D.V. Averin and K.K. Likharev, in: SQUID 85, eds. H.D. Hahlbohm and H. Lübbig (De Gruyter, Berlin, 1985).

[5] K.K. Likharev and A.B. Zorin, J. Low Temp. Phys. 59 (1985) 347;

D.V. Averin and K.K. Likharev, J. Low Temp. Phys. 62 (1986) 345; Sov. Phys. JETP 63 (1986) 427.

[6] F. Guinea and G. Schön, Europhys. Lett. 1 (1986) 585; J. Low Temp. Phys. 69 (1987) 219.

[7] K.K. Likharev, IEEE Trans. Magn. 23 (1987) 1138.

[8] D.V. Averin, Sov. J. Low Temp. Phys. 13 (1987) 208.

[9] M. Büttiker, Phys. Rev. B 36 (1987) 3548.

[10] K. Mullen, E. Ben-Jacob, R.C. Jaklevic and Z. Schuss, Phys. Rev. B 36 (1988) 98.

[11] M.P.A. Fisher, Phys. Rev. Lett. 60 (1988) 208.

[12] U. Geigenmüller and G. Schön, Physica $D$, to be published.

[13] E. Ben-Jacob, Y. Gefen, K. Mullen and Z. Schuss, Phys. Rev. B 37 (1988) 7400;

K. Mullen, E. Ben-Jacob and Y. Gefen, Physica D, to be published.

[14] Y. Gefen and D.J. Thouless, unpublished.

[15] K.K. Likharev, preprint.

[16] I. Giaever and H.R. Zeller, Phys. Rev. Lett. 20 (1968) 1504.

[17] J. Lambe and R. Jaklevic, Phys. Rev. Lett. 22 (1969) 1371.

[18] R.E. Cavicchi and R.H. Silsbee, Phys. Rev. Lett. 52 (1985) 1453.

[19] T.A. Fulton and G.J. Dolan, Phys. Rev. Lett. 59 (1987) 109.

[20] J.B. Barner and S.T. Ruggiero, Phys. Rev. Lett. 59 (1987) 807.

[21 ] L.S. Kuz'min and K.K. Likharev, JE'TP Lett. 45 (1987) 389.

[22] J.B. Barner and S.T. Ruggiero, Phys. Rev. B 36 (1987) 8870.

[23] M. Iansiti, A.T. Johnson, W.F. Smith, H. Rogolla, C.J. Lobb and M. Tinkham, Phys. Rev. Lett. 59 (1988) 489;

M. Iansiti, A.T. Johnson, C.J. Lobb and M. Tinkham, preprint.

[24] E. Ben-Jacob, F. Eamelas and K. Mullen, Josephson junctions: theory and applications, Lecture notes, University of Michigan, Ann Arbor (1988).

[25] M. Amman, E. Ben-Jacob, K. Mullen and G. Schön, unpublished.

[26] E. Shimshoni, S. Levit and Y. Gefen, unpublished.

[27] D.E. McCumber, J. Appl. Phys. 39 (1968) 3113; W.C. Stewart, Appl. Phys. Lett. 12 (1968) 277.

[28] H.D. Hahlbohm and H. Lübbig, eds., SQUID 80 (De Gruyter, Berlin, 1980); SQUID 85 (De Gruyter, Berlin, 1985), and references therein. 
[29] M. Buttiker, Phys. Rev. B 27 (1983) 6178; M. Buttiker and R. Landauer, Phys. Rev. Lett. 49 (1982) 1739; in: Advances in solid state physics, Vol. XXV. Festkörperprobleme, ed. P. Grosse (Vieweg, Braunschweig) p. 771; IBM J. Res. Dev. 30 (1986) 451; Phys. Scr. 32 (1985) 429.

[30] E. Ben-Jacob and Y. Imry, J. Appl. Phys. 52 (1981) 6806.

[31] A.C. Newell, in: Topics in modern physics. Solitons, eds. R. Bullough and P. Caudrey (Springer, Berlin, 1978).

[32] M. Buttiker and R. Landauer, in: Physics in one dimension, eds. J. Bernasconi and T. Schneider (Springer, Berlin, 1981).
[33] D. Bergman, E. Ben-Jacob, Y. Imry and K. Maki, Phys. Rev. A 27 (1983) 3345.

[34] P.M. Marcus and Y. Imry, Solid State Commun. 33 (1980) 345.

[35] G. Gruner, A. Zawadowski and P.M. Chaikin, Phys. Rev. Lett. 46 (1981) 511.

[36] E. Ben-Jacob, Solid State Commun. 47 (1983) 603.

[37] J. Bardeen, Phys. Rev. Lett. 45 (1980) 1978.

[38] K. Mullen, E. Ben-Jacob and Z. Schuss, Phys. Rev. Lett. 60 (1988) 1097. 